\title{
The Design and Implementation of Intelligent Campus Security Tracking System
}

\author{
Bhargavi Kaslikar ${ }^{1}$, Prof. Kirti Sawlani ${ }^{2}$ \\ '(Student, Pursuing masters in engineering in Electronics, /Department of Electronics, K.J.Somaiya college of \\ Engineering / Mumbai University, India) \\ ${ }_{2}^{2}$ (Assistant Professor, Department of Electronics, K.J.Somaiya college of Engineering / Mumbai University, \\ India)
}

\begin{abstract}
This paper implements Intelligent tracking system for campus security. The intelligent campus security tracking system is based on wireless communication services between nodes provided by RFID sensors and ZigBee. This system identifies the RFID tags within the region to prevent thefts and track the valuables, so as to protect the property of the stake holder on the campus. This system is based on radio frequency detection and IEEE 802.15.4 based ZigBee protocol. The tracking ability of RFID sensors and the ability of ZigBee to form a wireless sensor network has been effectively used in this paper to achieve the goal. RFID identifies the RFID tags on the Master and the slaves i.e valuables owned by the master, then sends the sensed information to $P C$ node through ZigBee. PC node generates corresponding warning depending on the result of match between the master and slave RFID tag information. When the warning occurs, its user can get the status of missing valuables, so the theft can be easily avoided.
\end{abstract}

Keywords: Microcontroller, RFID Tags, Security Tracking,Wireless sensor network,ZigBee

\section{Introduction}

The design of an intelligent campus security tracking system is based on RFID and ZigBee system which has full range of protection on campus. The main concern is to stop the increase in theft in colleges. This paper gives exhaustive study on IEEE 802.15.4 Based Intelligent Tracking System[1]. The intelligent campus security tracking system is based on wireless communication services between nodes provided by RFID sensors and ZigBee, and identifies the RFID tags within the region to prevent thefts and track the valuables, so as to protect the property of the teachers and students.

RFID tags are cheap, and can be reused. Data transmission is convenient and easy .ZigBee can be used to implement a wireless sensor network [2]. So by combining ZigBee and RFID technique we can achieve fast data transmission over a wide area by creating a wireless sensor network [3].

RFID sensor with ZigBee module will send the information of the read tags to the micro-controller. The micro- controller sends the data to wireless module to complete data transmission after simply data processing. ZigBee module provides two-way data transmission services and returns the corresponding control information obtained from micro-controller to PC for processing. The PC will give warning about the users and his valuables are valid or not.

\section{Block Diagram}

The whole system includes two levels design: hardware and software. Hardware includes interfacing of RFID readers, ZigBee wireless transmission module [4] and micro-controller. Each user will have specific Master RFID tag and slave RFID tags. While leaving the campus RFID module will scan the Slave and Master ID, if both the IDs matches then the campus door will open else system will prompt warning on mismatched RFID tags. 


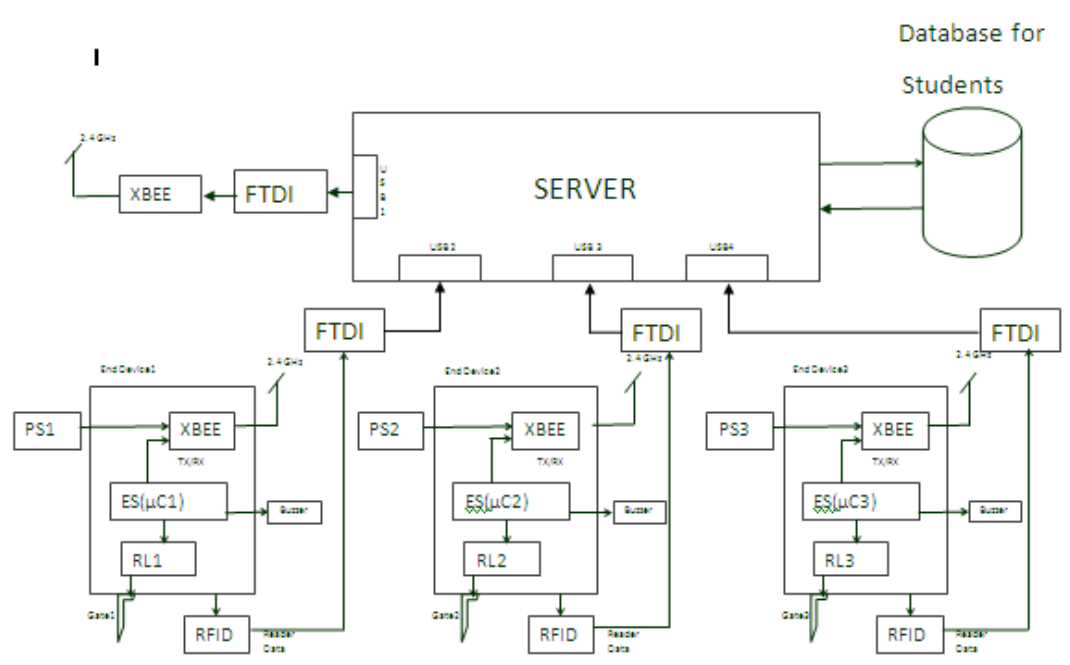

Fig1. Block diagram of Campus Security System.

Figure Fig1. Explains the block diagram of Security system. Entire campus will have three entry points. On each entry point (end devices) there will be a RFID reader Zigbee system and relays to open the door. RFID sensor is combined with ZigBee and a sensor node is formed.. Sensor node [5] will collect the data (scanned RFID tags) and will send the data to main server through FTDI (Future Technology Devices International).Server will do data processing for valid and invalid user and accordingly will generate warnings.

\section{Interfacing of ZigBee and RFID System}

Campus Security System will have three cards. The first card is Master unit. Master unit will have interfacing of ZigBee module and Max3232 .Basically this will be used to have communication between the server and ZigBee module. Figure Fig 2 gives the circuit details of Master unit used in campus security system. To provide a regulated dc supply to ZigBee Module voltage regulator is used. Master card is installed at Main entry gate of the campus.

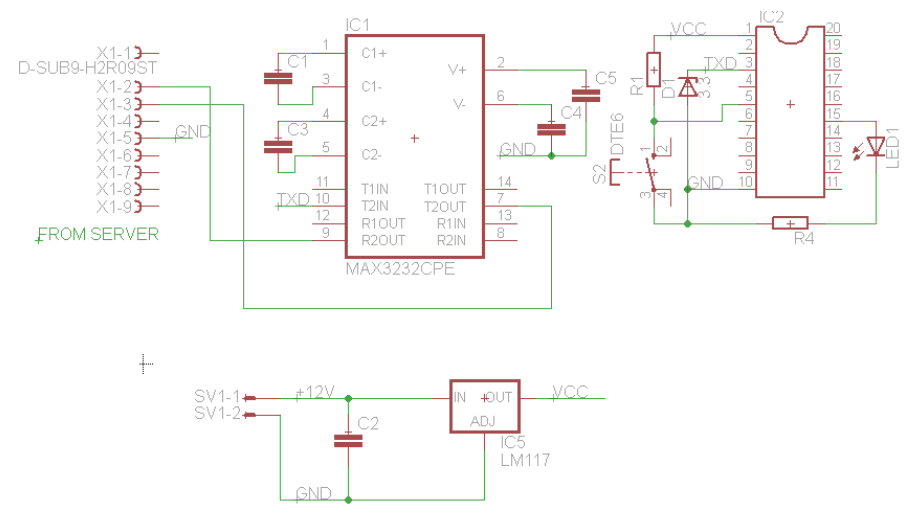

Fig2. Master unit of Campus Security Sysytem.

The second card which is interfaced with the system is Slave unit. Figure Fig 3 gives the circuit details of Slave unit used in campus security system. Slave unit will communicate between the ZigBee module, RFID reader and microcontroller. It is installed at all the entry points except main gate.To meet the voltage specifications of the microcontroller and ZigBee module different voltage regulators are used. 


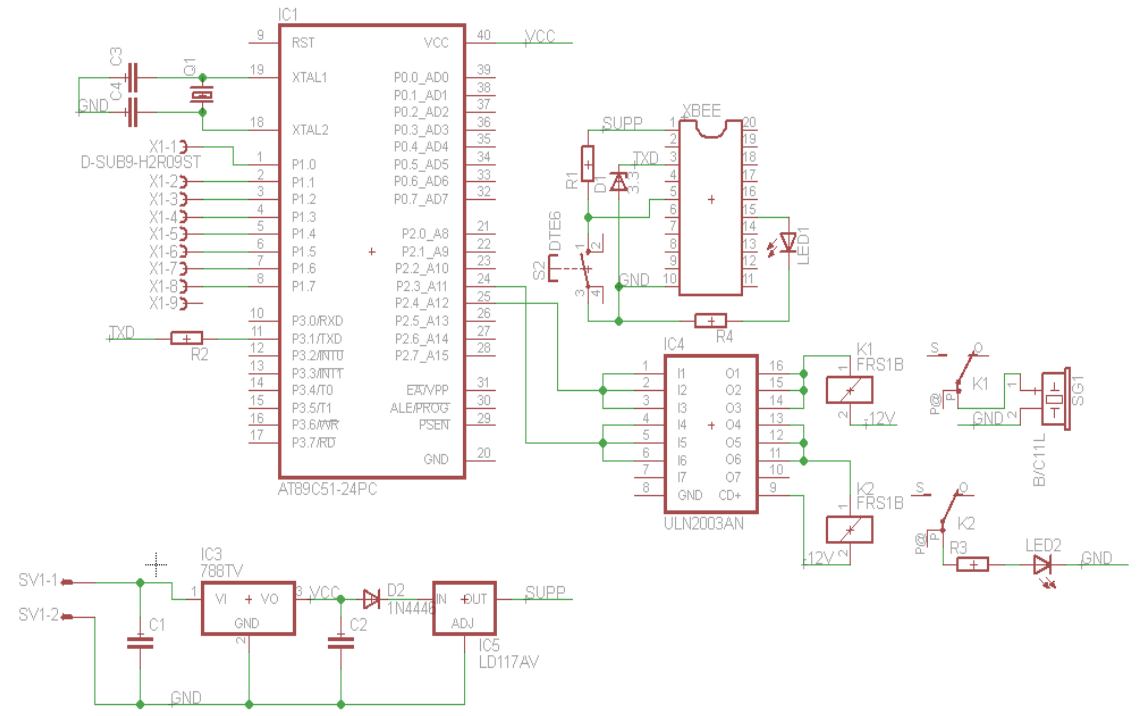

Fig3. Slave unit of Campus Security System.

RFID will scan the tags through ZigBee it is transmitted to the main server. After the data processing for valid and invalid access, microcontroller will operate the relay for closing and opening of the campus door. Figure Fig 4 elaborates the RFID module. Following are the cases which will describe the work flow of the system:

1) When master tag and slave label match, the system recognizes it as legal input.

2) When only tag appears, the system recognizes it as legal input.

3) When only tag appears, the system recognizes it as illegal input.

4) On every legal input the relay will switch to open the campus door.

5) On every illegal input the campus door will not open and warning alarm will be raised.

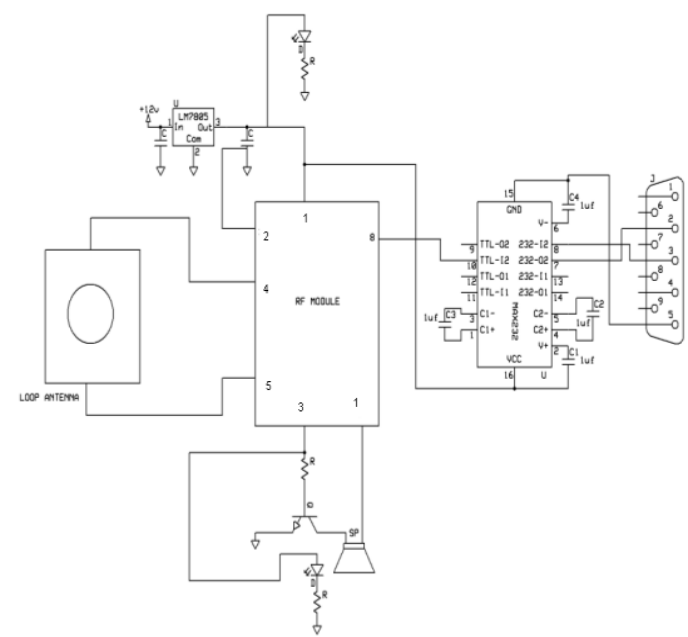

Fig4. RFID card of Campus Security System.

\section{Equations}

To get information about the area covered by the ZigBee, Power transmitted is calculated [6]. Effective transmit power is derived from adding the transmit antenna gain $\left(\mathrm{G}_{t}\right)$ to the total transmit power $\left(\mathrm{P}_{t}\right)$. Similarly, receiver antenna gain $\left(\mathrm{G}_{\mathrm{r}}\right)$ and receiver power $\left(\mathrm{P}_{\mathrm{r}}\right)$ can be derived from the following equation:

Where: $\mathrm{P}_{\mathrm{r}}=$ Receiver power in $\mathrm{dBm}$

$$
\mathrm{P}_{\mathrm{r}}=\mathrm{P}_{\mathrm{t}}+\mathrm{G}_{\mathrm{r}}+\mathrm{G}_{\mathrm{t}}+\mathrm{L}
$$

$\mathrm{P}_{\mathrm{t}}=$ Transmitter power in $\mathrm{dBm}$

$\mathrm{G}_{\mathrm{r}}=$ Receiver antenna gain in $\mathrm{dB}$

$\mathrm{G}_{\mathrm{t}}=$ Transmitter antenna gain in $\mathrm{dB}$

$\mathrm{L}=$ Attenuation at $2450 \mathrm{MHz}$ in $\mathrm{dB}$

Assuming the antenna gain for both transmitter and receiver is $0 \mathrm{~dB}$, receive power is given by: 
for free-space propagation conditions:

and for indoor propagation conditions

$$
\mathrm{P}_{\mathrm{r}}=\mathrm{P}_{\mathrm{t}}-(33 \log (\mathrm{d} / 8)+58.5)
$$

$$
\mathrm{P}_{\mathrm{r}}=\mathrm{P}_{\mathrm{t}}-(40 \log \mathrm{d}+50.3)
$$

\section{Experimental Results}

Simulation results are shown in figure Fig 5. And Fig 6. Fig 5 shows the indication of swiping the RFID tag by displaying unique card ID.

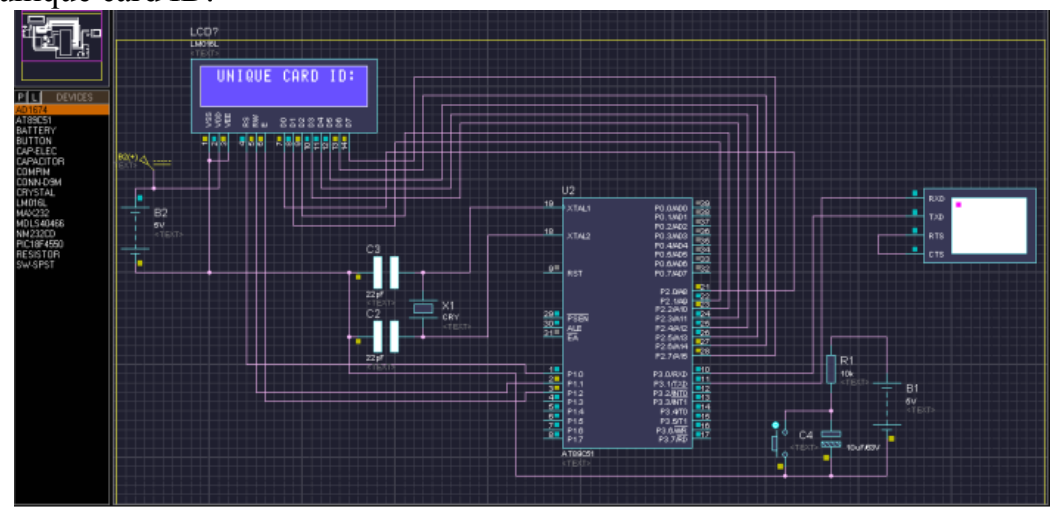

Fig 5. Motion contours are detected and rectangle box is drawn around each of them.

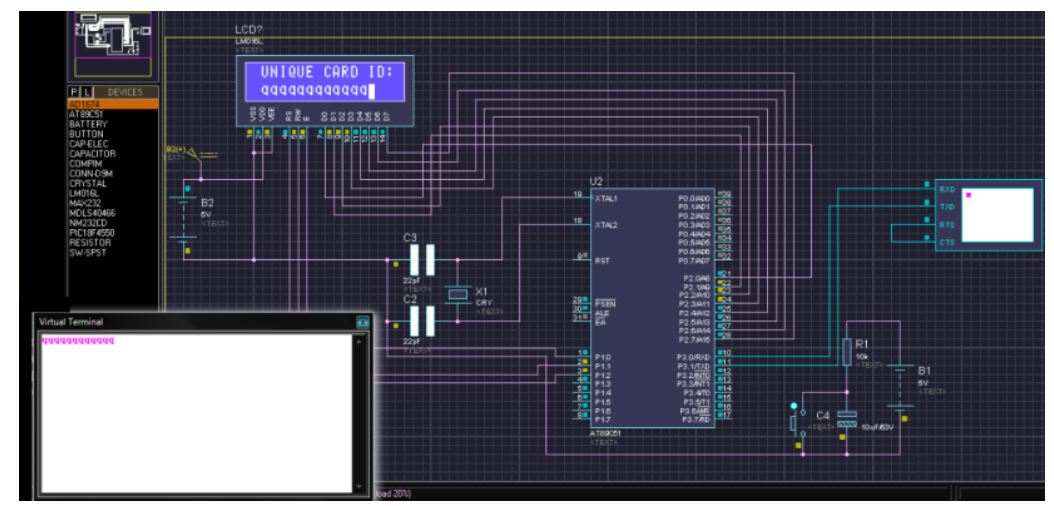

Fig 6 shows the result of swiping the RFID tag by displaying card ID details i.e RFID tag details

\section{Conclusion}

We proposed an idea how RFID and ZigBee can be interfaced with microcontroller to handle the security issues in a campus. The system authenticates the user information by obtaining the swiped RFID details. A passive RFID tag makes the system cost effective and ZigBee implementation helps in covering wide area. In this way valuables of an individual can be protected.

\section{Future Work}

After implementing RFID protocol, we observed that we need to maintain a complete database which will indicate all the transactions like how many times only the master or master along with the slave has used the system. Also we can track the number of times invalid users have tried to cross the system. In this way we can completely protect the wide campus.

\section{References}

[1] Ying Chen,Yuntao Wang,Xiaokang Li,Li Gao,The Design and Implementation of Intelligent Campus Security Tracking System Based on RFID and ZigBee,978-1-4244-9439-2/11 C2011 IEEE.

[2] Waltenegus Dargie,Fundamentals of Wireless Sensor Networks: Theory and Practice Christian Poellabauer 47-68

[3] Drew Gislason, Zigbee Wireless Networking 151-154

[4] Wei Wang, Guangyu He and Junli Wan, Research on Zigbee wireless communication technology The National Natural Science Foundation of China ( No. 51077079),978-1-4244-8165-1/11 C2011 IEEE

[5] Freescale IEEE 802.15.4 / ZigBee Node RF Evaluation and Test Guidelines Reference Manual, Document Number: ZRFETRM Rev. $1.005 / 2010$

[6] Shah, P., Vishwakarma Inst. of Technol., Pune, Shaikh, T., Ghan, K., Shilaskar, S.,Power Management Using ZigBee Wireless Sensor Network,Emerging Trends in Engineering and Technology, 2008. ICETET ' 08. 